\title{
SUBJECT INDEX*
}

$21 \mathrm{~cm}$ radio line, 216

absorption, 3

free-free, 157

active galactic nuclei, 333,422

low luminosity, 345

precursors, 345

unified scheme, 345

airglow, 257

time variability, 257

A IV surveys, 456

albedo, 199, 210, 239, 257, 485

for single scattering, 127

Alfvén velocity, 414

angular correlation function, 369

anisotropies, 396, 400

dipole, 283, 295

quadrupole, 283

large-scale and small-scale, 400

aperture synthesis, 283

Apollo, 17, 227

Apollo-Soyuz Test Project, 227

asymmetry, 127, 199, 239

atmospherically scattered light, 75

atomic hydrogen, 157

autocorrelation function, 408

background, 307

$1400-1900 \AA, 295$

distance, 3

radiation, $21,295,420$

starlight, 105

balloon-borne telescopes, 35

Balmer line emission, 107, 157

baryon isocurvature model, 269, 295

Be band, 241

Berkeley bright patches, 227

Berkeley-Nagoya balloon experiment, 469

biasing, 295

big bang, 3

hot, 469

model, 469

BN-type objects, 113

branching ratio, 387

bright bulge, 35, 203, 205

C+ emission from the galaxy, 149

calibration, 118

absolute, 203
CCD

camera, 283

imaging, 210

mosaic maps, 357

surveys, 245

chromospherically, 107

cirrus, 157, 235, 465

high latitude, 216, 235

infrared, 139

cloud(s)

heating, 216

high velocity, 157

small optically thin, 241

c-models, 269

CO maps, 35

coherence length, 295

cold dark matter, biased, 269

color excesses E(B-V) or E(U-B), 375

color index $\mathrm{B}-\mathrm{V}, 375$

color-magnitude relation, 269

column density of the $H^{+}, 157$

Comptonization, 317

cooling flows, 283, 414

coronal component, 157

cosecant law, 257

Cosmic Background Explorer, 149, 317, 381, 469

cosmic background radiation, 283, 295, 400, 435

cosmic microwave background, 392, 406

temperature, 390

cosmic ray heating, 63

cosmic rays, 157, 414

cosmology, 369, 420

models, 257, 469

count(s)

probability distribution, 408

faint galaxies, 365, 469

optical counts of galaxies, 469

sources, 469

cryogenic telescope, 435

Cygnus, 157

dark

clouds, 199, 239

method, 257

prominent nearby, 205

count, 227

mass, 295

*Page numbers in the index refer to the first page of the article that discusses the topic. 
matter, 283, 357

nebulae, 127

decay photon spectrum, 387

decaying particles, 387

deceleration parameter, 269, 365

density, 157

fluctuation, 418

baryonic matter, 345

baryonic parameters, 295, 345

local galaxy, 269

local low-density region, 241

profile, 239

structure, 239

diffuse, 307

emission, 157

extreme-UV flux, 157

extragalactic background, 149

Galactic emission, 406, 459

intergalactic light, 357

interstellar medium, 157

ionized gas, 157

infrared radiation, 435

light in clusters of galaxies, 461

optical line emission, 157

radio emission, 406

scattered starlight, 257

ultraviolet background, 225, 227, 449

ultraviolet radiation at the galactic pole, 225

diffuse Galactic light, 21, 127, 239, 257, 449

models, 127, 239

observations in the ultraviolet, 127

polarization, 75

disk, 205

inner thin, 35

maximum radius, 121

outer, 35

thick, 85, 121

discrete source contribution, 345

distance determination, 199

dust, 118, 225, 227, 237, 375, 402

albedo, 127

central dust lane, 205

clouds, 239

clouds, intergalactic, 245, 385

cosmic, 435

emission, 35

warm dust emission, 113

high latitude clouds, 357

interplanetary, 218

interstellar, 75, 139, 199, 210, 214, 225, 229, 396,459

intergalactic, 245, 283 intragroup, 375

local interstellar, 203

mass, 239

scattering, 441

spectrum of high galactic latitude dust, 396

-y galaxy, 283

dynamics, 231

Dynamics Explorer 1, 225

early universe, 387, 398, 435

electron

column density, 157

temperature, 15, 7

element production, 295

emission, 345

at north Galactic pole, 396

line emission, 157

from external galaxies, 149

-line background, 157

measure, 157

nebulae, 459

optical line, 157

energy density, 345

epoch of last scattering, 295

evolution of galaxies, 365

evolution, radio, 469

explosion picture, 295

extinction, 35

coefficient, 367

mapping, 199, 239

extragalactic, 229, 307

extragalactic background radiation, 21, 223, 225 ,

$257,269,365,400,461,469$

from quasi-stellar objects, 269

extragalactic $\gamma$-ray background, 345

extragalactic X-ray background, 333, 345

extreme ultraviolet, 157

background, 463

Fabry-Perot observations, spectroscopy, 157

Far Ultraviolet Imaging Spectrograph, 459

far ultraviolet background, 157, 171, 441

far-infrared, 149

Fermi acceleration, second-order, 414

filling fraction, 157

fine-structure line emission, 149, 157

flare, 107

fluctuations, 283, 295, 357, 402, 416, 469

smoothness of the sky at $2.2 \mu \mathrm{m}, 317$

forbidden lines, 157

forward-scattering, 127

Galactic

arms, 123

component, 225 
corona, 441

disk, 35

dynamics, 35

extinction, 367

$H \propto$ background, 157

halo, 157, 371

latitude dependence, 225

models, 35

radiation field, 157

three-dimensional model, 35

windows, 396

galactic background, 139

infrared radiation, 113, 435

polarization, 75

Galactic structure, 21, 35, 110, 121, 231, 233, 422, 435

Eridanus, 157, 235

Orion arm, 157

Orion-Eridanus shell, 157

Perseus spiral arm, 157

Sagittarius arm, 157

galaxies, 245, 373

luminous infrared, 345

merged catalogue, 367

metacatalogue, 367

normal, 345

primeval, 245, 269, 317

strong radio, 345

very young, 257

young, 345, 269, 295

galaxies, clusters of, 283, 333, 345, 377

Abell 2319, 406

Abell 2029, 357

Abell 426, 371

Virgo, 373, 379

galaxies, individual

M31, 85, 118

NGC 13257, 377

NGC 4151, 345

NGC 5033, 85

NGC 891, 85

galaxy

clustering inhomogeneities, 269

counts, 269, 295

distribution, 245

evolution, 245, 257

formation, 257, 420

frames of reference, 295

topology, 245

two-point spatial correlation function, 295

gamma-ray background, 469

gas hot diffuse intergalactic, 345

hot intracluster, 345

ionized, 149, 157

ionized interstellar, 157

optically emitting, 157

temperature, 157

gas-dust offsets, 235

gas-to-dust ratios, 235

GEISHA, 118

geocoronal emission, 157

geocoronal $H \alpha$ and $H \beta, 157$

global parameters, 35, 205

globular clusters, 377

gravitational lens, 245,418

great attractor, 295

groups, 375

Gum nebula, 157

$\mathrm{H} \alpha$

filaments, 235

line, 107

photography, 157

$\mathrm{H}_{2}$ flourescence, 441

H $\beta 157$

HAC luminescence, 210

halo, 315,357

hard X-ray

background, 469

spectra, 345

HEAO-1 A4, 406

heating, photoelectric, 63

heavy element production, 295

helios, 75, 105

Herschel, William, 85

high resolution, 205

high spectral resolution, 157

highly ionized species, 149

$\mathrm{H}$ II regions, 149, 157

compact, 113

Hopkins Ultraviolet Background Experiment, 441

Hubble

constant, 85, 295

length, 295

type of the Galaxy, 85

inflation, 295

infrared, 123, 139, 157, 231, 269

background, 381, 387, 406, 429

cirrus, 139

emission, 157, 385

extragalactic background light, 317

point source sky, 110

radiation, 35

sky brightness, 295 
spectral line observations, 149

telescope, 317, 435

Infrared Astronomical Satellite (IRAS), 118, 218,

$121,123,235,465$

$100 \mu \mathrm{m}$ cirrus, 210

Explanatory Supplement, 118

faint point sources, 373

flukes, 113

galaxy counts, 269

sky flux maps, 214

source counts, 110

Infrared Space Observatory (ISO), 149, 429

intergalactic light in clusters, 283

intercluster medium, 414

intergalactic gas, 257,469

two-phase model, 345

reheating, 345

intergalactic $\mathrm{H}$ I cloud, 157

intergalactic ionized hydrogen, 257

intergalactic medium, 424, 441

interstellar, 237

$\mathrm{CN}, 392$

extinction, 157

grains, 127

$\mathrm{H}_{2}$ emission, 459

$\mathrm{H} \mathrm{I}, 235$

ionization, 157

matter, 139

interaction of the ISRF with, 63

physics, $\mathbf{4 3 5}$

polarization, 75

interstellar medium

dust, 229

hot, 459

interstellar radiation field, 485

in central region of the Galaxy, 63

in galactic disk, 63

in solar vicinity, 63

inside molecular clouds, 63

mean radiation intensity, 63

by dust, 63

by stars, 63

variation with galactocentric distance, 63

inverse Compton, 283, 387

ionization ratio, 157

ionization source, 157

ionization state, 157

ionizing photon flux, 157

infrared

filaments, 235

galaxies, 381

radiation, 118 starburst population, 269

telescope, 317, 435

isotropy, $283,333,345$

component, 225

Johns Hopkins Applied Physics Laboratory, 441

$\mathrm{K}$-band polarization measurements, 35

K-corrections, 269

kinematics, 157

Landolt, 357

large-scale structure, 369,416

large-field technique, 127

latitude dependence, 225

light of night sky, 257, 461

lines

C II, 435

O I, 435

line of sight, 3

line profile, 390

local interstellar medium, 463

luminescence, 233

luminosity

density, 257

evolution, 269, 365

function, 107, 110, 269

$12 \mu \mathrm{m}, 121$

Lyman break, 245

Lyman continuum photons, radiation, 157

Lyman limit systems, 269

magnetic field, 414

in clusters of galaxies, 406

primeval, 402

main body, 205

mapping of the galactic plane, 435

mass

autocorrelation function, 295

large-scale fluctuations, 295

missing, 283

mass-to-light ratio, 283, 357

measurement, absolute, 149

mergers, 269

MgI doublet, 257

MHD wave turbulence, 414

microchannel plate detectors, 441

microwave background, 333, 387, 398, 422, 469

Milky Way, 85, 100, 118, 212

polarization, 75

Mira variables, 118

missions, future, 456

molecular clouds, 465

molecular hydrogen fluorescence, 441

multivariate analysis, 214

$\mu$-models, 269 
Nagoya-Berkeley rocket experiment, 317 near-infrared, 35

near-infrared background radiation, 283, 435

near-infrared maps, 35

nebulae

Coalsack, 239

L134, 357, 257

L1642, 257

nebulae, reflection, 210,459

nebular lines, 157

neutral hydrogen, 149, 157, 225

neutrinos, massive, 283, 387

night sky, 157

night sky level, 357

nonthermal motions, 157

nonthermal particles, 414

north ecliptic pole, 105

number evolution, 365

obscuration, 3

Olbers's paradox, 3, 257, 269

opaque filter, 463

optical, 269

optical background, 295, 469

optical brightness of the sky, 367

optical scattering, 210

Orbiting Astronomical Observatory 2, 127

OSO-5, 75

Owens Valley Radio Observatory, 295

particle accelerations, 414

particle background, 463

Petscheck reconnection, 414

phase function, 127, 199, 210

phase space, 283

photodissociation regions, 149

photoionization, 157

photoionization model, 469

photoionizing radiation, 469

photometric measurements, 157

photometric observations, 157

photon-counting image system, 449

Pioneer 10, 75, 85, 105, 127, 257

pixel distribution function, 357

plasma

high temperature, 241

hot intergalactic, 424

point spread function, 357

polarization, 333

atmospherically scattered light, $\mathbf{7 5}$

circular, 75

diffuse galactic light, 75

effects, $\mathbf{4 0 2}$

linear, 75 polycyclic aromatic hydrocarbons, 212

protogalaxies, 245

pulsar dispersion measures, 157

quadrupole moment, 295

quasars, 345

$3 \mathrm{C} \mathrm{273,} 345$

flat radia spectra, 345

luminosity, 418

radio-quiet, 345

steep radio spectra, 345

radiation field, 210

local, 157

radiative decay, 283

radio background emission, 469

radio free-free absorption, 157

red fluorescence, 485

reddening, 257

redshift asymmetry, 375

redshift distribution, 269, 375

redshift of galaxies, 245

relativistic beams, 345

relic, 295

ROSAT, 463, 465

Sachs-Wolfe effect, 295

Saltpeter initial mass function, 269

scalar velocity autocorrelation function, 295

scale height, 157

scale length, 85

scattering, 75, 225, 227, 229, 231

efficiency of interstellar dust grains, 379

properties, 127

Schechter luminosity function, 269

Science Applications Intl. Corp., 441

Seyfert 1 nuclei, 345

shock activity, 235

shocks, 157

S II, 157

Small Explorer program, 441

small-field technique, 127

soft X-ray, 157, 241

soft X-ray/XUV background, 463, 465

source, 123

source number-flux distribution, 408

Space Infrared Telescope Facility, 149

Space Shuttle, 227

Space Station, 441

Spacelab-2 Infrared Telescope, 35

Spartan payload, 459

spectra, 345

synthetic, 257

spectrophotometric evolution model, 365

spectroscopic observations, 157 
spectrum, 35, 333

of mass fluctuations, 295

of the extragalactic $\mathrm{X}$ - and $\boldsymbol{\gamma}$-ray backgrounds, 345

disks, spiral galaxy, 245

star bursts, 412

star counts, 103

automated, 199

star count model, 121

star formation, 235,412

massive, 113

prominent regions, 205

star masks, 257

starlight, integrated, 21, 105, 257

brightness and colors, 103

stars

active, 107

distribution of old, evolved stars, 35

$\mathrm{dMe}, 107$

first generation, 283, 435

HD 21483, 392

hot, evolved, 157

$\mathrm{K}$ - and M-giant, 35

nearest, 3

$O$ and $B, 157$

$\mathrm{OH} / \mathrm{IR}, 118$

population III, 469

red dwarfs, 107

red giants, 118

SN 1987A, 387

stellar population, 35,85

stellar wind bubbles, 459

structure (see also Galactic structure)

structure, three-dimensional, 231

structure, very broad, 233

structures, very large-scale, 422

submillimeter, 400

background, 295, 387

excess, 317,381

Submillimeter Wavelength Astronomical

Satellite, 149

superbubble, 235

supernova remnants, 459

surface brightness, 85, 199, 205, 239

distribution, 35, 203

fluctuations, 345

surface photometry, 100, 239

TD-1 satellite, 127

temperature, 157

and nonthermal motions, 157

thermal bremsstrahlung, 424

thermal emission, 231 thermal radiation from dust, 295

thermal reemission, 283

thermal reradiation, 210

Thomson scattering, 295

tidal stripping, 357

U-B, 375

UBVRI photometry, 105

ultraviolet, 229, 269, 307

ultraviolet background, 223, 469

correlation with H I $21 \mathrm{~cm}$ radio intensity, 379

ultraviolet extinction, 233

ultraviolet Ceti, 107

universe, expanding, 3

universe, static, 3

universe, steady-state, 3

University of Wisconsin, 157

U. S. Air Force Space Test Program, 459

ultraviolet and $\mathrm{X}$-radiation, 469

UVX experiment, 227

vacuum ultraviolet background radiation, 379

velocity field, large-scale, 295

velocity, peculiar, 295

visible, 157,365

visible continuum, 157

visible universe, 3

Wide Field Camera, 463, 465

winds, hot galactic, 345

X-ray background, 333, 412, 416, 422, 469, 295

$345,408,424$

and hot intergalactic medium, 345

residual, 345

$\mathrm{X}$-ray binaries, 412

massive, 345

X-ray extragalactic background, 422

$X$-ray shadowing, 465

$\mathrm{X}$-ray spectra, 345

X-Ray Telescope (XRT), 465

Zel'dovich spectrum $P_{\mathrm{K}} \alpha$ K, 295

zero-point field, 424

zodiacal cloud, 231

zodiacal emission, 118, 218

zodiacal light, 75, 214, 257, 441

polarization, 75

Zwicky magnitude, 367 